\title{
Aperçu des éclosions d'origine alimentaire au Canada signalées par l'entremise du Registre fédéral des éclosions de 2008 à 2014
}

\author{
Bélanger $\mathrm{P}^{1 *}$, Tanguay $\mathrm{F}^{1}$, Hamel $\mathrm{M}^{1}$, Phypers $\mathrm{M}^{1}$ \\ ${ }^{1}$ Centre des maladies infectieuses d'origine alimentaire, environnementale et zoonotique, Agence de la santé publique \\ du Canada, Ottawa (Ontario) \\ *Correspondance : philippe.belanger@phac-aspc.gc.ca
}

\section{Résumé}

Contexte : Au Canada, des enquêtes sur l'éclosion des maladies entériques sont menées à l'échelle locale, provinciale/territoriale et fédérale. Par le passé, la surveillance de routine des éclosions n'était pas effectuée dans toutes les provinces et les territoires. Pour combler cette lacune, l'Agence de la santé publique du Canada, en association avec les autorités de santé publique des provinces et des territoires, a développé le Registre fédéral des éclosions, un système de déclaration en ligne sécurisé.

Objectif : L'analyse visait à décrire les enquêtes sur les éclosions d'origine alimentaire signalées par l'intermédiaire du Registre fédéral des éclosions entre 2008 et 2014.

Méthodologie : Les rapports complétés sur les enquêtes menées entre 2008 et 2014 pour toutes les autorités de santé publiques participantes au Canada ont été extraits et une analyse descriptive des éclosions d'origine alimentaire portant sur les agents étiologiques, la gravité de la maladie, la durée de l'éclosion, le milieu d'exposition et la source de l'éclosion a été effectuée.

Résultats : Au total, 115 éclosions d'origine alimentaire ont été signalées et incluses dans la présente analyse. Cela représente $11,2 \%$ de toutes les éclosions signalées dans le module sur les maladies entériques du Registre fédéral des éclosions entre 2008 et 2014. Les infections à Salmonella ont été la cause d'éclosion d'origine alimentaire la plus fréquemment signalée (40,9\%) et Enteritidis a été le sérotype le plus fréquemment signalé. Les éclosions d'origine alimentaire représentaient 3301 cas de maladies, 225 hospitalisations et 30 décès. Dans l'ensemble, 38,3\% des éclosions d'origine alimentaire signalées sont survenues dans la collectivité et 32,2\% ont été associées à un établissement de services alimentaires. La plupart des enquêtes sur les éclosions d'origine alimentaire $(63,5 \%)$ ont associé un aliment précis à l'éclosion, le plus fréquent étant la viande.

Conclusion : Le Registre fédéral des éclosions favorise l'échange de renseignements et la collaboration entre les partenaires du domaine de la santé publique au Canada. De plus, il permet de dresser un portrait à l'échelle nationale des éclosions d'origine alimentaire. Cette analyse démontre l'utilité des données tirées du Registre fédéral des éclosions en tant que source d'information pour décrire les enquêtes sur les éclosions d'origine alimentaire au Canada.

\section{Introduction}

On estime qu'environ quatre millions d'épisodes de maladies d'origine alimentaire contractées au pays surviennent chaque année au Canada (1). Certaines de ces maladies peuvent entraîner une hospitalisation et même la mort. Des estimations récentes indiquent que chaque année au Canada, 11600 hospitalisations et 238 décès sont associés à des maladies d'origine alimentaire contractées au pays (2). Les maladies d'origine alimentaire sont investiguées pour de nombreuses raisons, notamment pour cerner et éliminer la 
source de l'éclosion et empêcher l'apparition de nouveaux cas. De plus, les résultats des enquêtes peuvent mener à des recommandations visant à éviter les éclosions dans l'avenir (3).

Au Canada, des enquêtes sur l'éclosion des maladies entériques sont menées à l'échelle locale, provinciale/territoriale et fédérale. Par le passé, la surveillance de routine des éclosions n'était pas effectuée dans de nombreuses autorités de santé publique canadiennes. Pour pallier à ce manque de renseignements et répondre à la nécessité de créer un système de surveillance des éclosions à l'échelle nationale, l'Agence de la santé publique du Canada (ASPC), en association avec les autorités de santé publique des provinces et des territoires, a inauguré en 2008 le Registre fédéral des éclosions.

Ce système a été conçu comme un outil de surveillance nationale, et toutes les données qu'il contient sont accessibles aux autorités de santé publique participantes. Divers professionnels de la santé publique à l'échelle locale, provinciale/territoriale et fédérale peuvent consulter, compiler les données et produire des rapports sur les éclosions signalées par l'entremise du Registre fédéral des éclosions. Ces renseignements peuvent servir à guider les enquêtes sur les éclosions en cours en fournissant des données historiques sur les éclosions de certains agents pathogènes dans le but de générer des hypothèses sur l'étiologie des éclosions actuelles et pour orienter les interventions en santé publique. Le système favorise également l'échange de renseignements et la collaboration entre les nombreux partenaires du domaine de la santé publique au Canada.

L'objectif du présent article est de décrire les enquêtes sur les éclosions d'origine alimentaire menées par les provinces participantes entre 2008 et 2014.

\section{Méthodologie}

\section{Sources des données}

Le Registre fédéral des éclosions est une application en ligne sécuritaire qui peut être utilisée par les professionnels de la santé publique à l'échelle locale, provinciale/territoriale et fédérale pour résumer et mettre en commun les résultats des enquêtes sur les éclosions dans un format normalisé. Le Réseau canadien de renseignements sur la santé publique (RCRSP) fournit la plateforme qui héberge le Registre fédéral des éclosions. Le système comporte actuellement deux modules : un pour les maladies entériques et d'origine hydrique et alimentaire, et un autre pour les maladies respiratoires et les maladies évitables par la vaccination. À l'heure actuelle, six provinces (Colombie-Britannique, Manitoba, Ontario, Nouvelle-Écosse, Île-du-Prince-Édouard et Terre-Neuve-et-Labrador) ainsi que le Centre des maladies infectieuses d'origine alimentaire, environnementale et zoonotique (CMIAEZ) de l'Agence ont graduellement mis en application le module sur les maladies entériques du Registre fédéral des éclosions et signalent systématiquement les éclosions. Chaque autorité de santé publique saisit des données dans le Registre fédéral des éclosions et chaque province établit ses propres lignes directrices relativement au signalement des éclosions. L'Agence signale quant à elle les éclosions d'ampleur multijuridictionnelle qui sont sous sa responsabilité.

Les rapports finaux sur les enquêtes menées entre 2008 et 2014 pour toutes les autorités de santé publique participantes au Canada ont été extraits le 7 mai 2015 à partir du module sur les maladies entériques et d'origine hydrique et alimentaire du Registre fédéral des éclosions.

\section{Analyse des données}

Le présent relevé offre une analyse descriptive des agents étiologiques, de la gravité de la maladie, de la durée de l'éclosion, de la source de l'éclosion et du milieu d'exposition. L'éclosion au sein d'une collectivité et l'éclosion au sein d'un établissement ont été définies conformément au texte de l'encadré ci-dessous : 


\section{Définition d'une éclosion au sein d'une collectivité et d'une éclosion au sein d'un établissement pour le Registre fédéral des éclosions}

Éclosion au sein d'une collectivité : deux ou plusieurs cas apparemment non liés d'une maladie similaire pouvant être liés épidémiologiquement entre eux (c.-à-d. associés en fonction du temps, et/ou lieu et/ou de l'exposition).

Éclosion au sein d'un établissement : au moins trois cas d'une maladie similaire pouvant être liés épidémiologiquement (c.-à-d. associés en fonction de l'exposition, au cours d'une période de quatre jours dans un milieu institutionnel).

Les données ont été revues avant de procéder à l'analyse afin de déceler toute incohérence et redondance. Les éclosions de Clostridium difficile et d'entérocoques résistants à la vancomycine (ERV) n'ont pas été inclues puisqu'il s'agit généralement d'infections associées aux soins de santé, dont la prise en charge peut différer grandement de celle des éclosions de maladies entériques. Les résumés en double, c'est-à-dire les situations où au moins deux paliers de compétence distincts (p. ex. l'Agence et une province, une province et une unité de services de santé ou une régie régionale de la santé) ont signalé une enquête conjointe sur une éclosion, ont aussi été éliminés de l'analyse. Dans ces situations, le rapport de l'autorité de santé publique responsable de l'éclosion a été retenu aux fins d'analyse et les autres rapports ont été exclus.

L'année d'enquête utilisée pour cette analyse a été établie à partir de la date de début de l'enquête sur l'éclosion. Une analyse descriptive de l'emplacement géographique des cas, des principaux agents étiologiques, du nombre de cas confirmés en laboratoire et de cas cliniques, de la gravité de la maladie et des milieux d'exposition et de transmission a été effectuée. De plus, le nombre de cas, d'hospitalisations et de décès par agent étiologique a été déterminé. La durée de l'éclosion a été déterminée et calculée en fonction de la différence entre les dates d'apparition des symptômes du premier et du dernier cas signalés. Les rapports qui n'indiquaient aucune valeur dans l'un ou l'autre de ces champs ont été exclus. On a également procédé à des analyses plus ciblées des milieux de transmission par agent étiologique ainsi que sur les sources identifiées lors d'éclosions d'origine alimentaire.

Les analyses ont été effectuées dans Microsoft Excel 2010.

\section{Résultats}

Entre 2008 et 2014, 1027 éclosions ont été saisies dans le Registre fédéral des éclosions. De ce nombre, 115 étaient des éclosions d'origine alimentaire et ont été incluses dans cette analyse (Figure 1). Ces éclosions d'origine alimentaire représentent $11,2 \%$ de toutes les éclosions signalées dans le Registre fédéral des éclosions entre 2008 et 2014. Elles sont donc, après la contamination interhumaine, le mode de transmission le plus fréquent pour les éclosions de maladies entériques signalées. Les autres modes de transmission comprenaient la transmission d'origine hydrique et environnementale ou d'un animal à une personne, ainsi que les modes de transmission multiples. 
Figure 1 : Proportion des enquêtes signalées par l'entremise du Registre fédéral des éclosions selon le mode de transmission, de 2008 à 2014 ( $n=1$ 027)

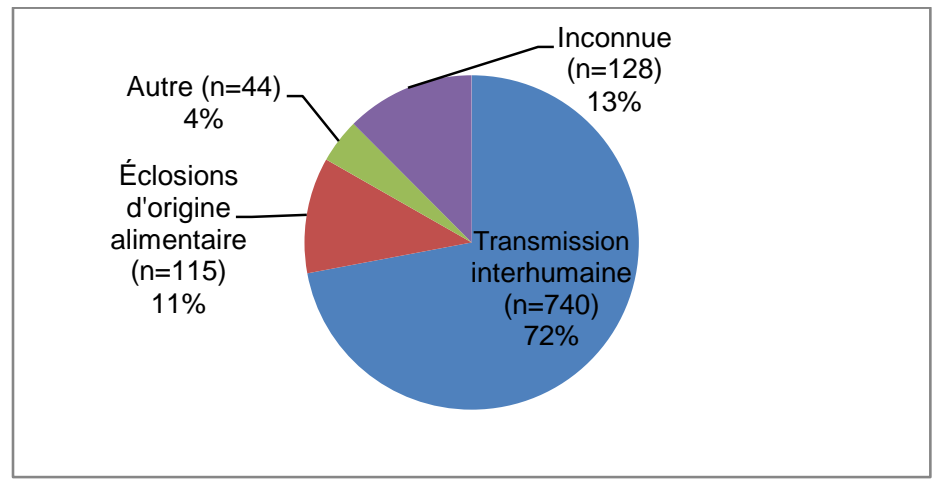

Les éclosions d'origine alimentaire ont été le mode de transmission le plus fréquemment signalé dans les éclosions rapportées par l'Agence (37 cas sur 46, soit $80,4 \%$ des cas). Plus de la moitié des éclosions d'origine alimentaire dans le Registre fédéral des éclosions ont été signalées par la Colombie-Britannique (67 cas sur 115 , soit $58,3 \%$ des cas).

\section{Agents étiologiques}

Le Tableau 1 présente les agents étiologiques associés aux éclosions d'origine alimentaire signalées dans le Registre fédéral des éclosions. Parmi les 115 éclosions d'origine alimentaire signalées entre 2008 et 2014, un agent étiologique a été précisé dans 106 cas (92,2\%). La majorité des éclosions (73\%) ont été attribuées à des agents bactériens, tandis que les éclosions d'étiologie virale représentaient $14,8 \%$ des cas. Les agents étiologiques les plus souvent mis en cause étaient les bactéries Salmonella $(40,9 \%)$ et Escherichia (14,8\%) ainsi que le norovirus (12,2\%). Un sérotype a été signalé dans 46 des 47 éclosions à Salmonella. Le sérotype Enteritidis a été le sérotype le plus fréquemment signalé et a été mis en cause dans 22 des 47 cas d'éclosions à Salmonella (46,8 \%). Toutes les éclosions à bactérie Escherichia ont été attribuées à $E$. coli $\mathrm{O} 157$ producteur de vérotoxine (ECVT).

Au total, 3301 cas ( 2261 cas confirmés en laboratoire et 1040 cas cliniques) ont été associés aux 115 enquêtes sur les éclosions d'origine alimentaire. Dans l'ensemble des cas, la plus grande proportion a été associée aux infections à Salmonella (2 041 cas sur 3 301, soit $61,8 \%$ des cas, desquels $87 \%$ ont été confirmés en laboratoire). La proportion qui arrive au deuxième rang est celle du norovirus (352 cas sur 3301 des cas, soit 10,7 \%) dont la grande majorité (92,9\%) étaient des cas cliniques. 
Tableau 1 : Nombre d'enquêtes sur les éclosions d'origine alimentaire signalées par l'entremise du Registre fédéral des éclosions et nombre total de cas selon l’agent étiologique, de 2008 à 2014 ( $n=115)$

\begin{tabular}{|c|c|c|c|c|}
\hline \multirow[b]{2}{*}{ Agent étiologique } & \multirow[b]{2}{*}{$\begin{array}{l}\text { Éclosions } \\
\mathrm{n}(\%)\end{array}$} & \multirow[b]{2}{*}{$\begin{array}{c}\text { Nombre total } \\
\text { de cas } \\
\mathrm{n}(\%)\end{array}$} & \multicolumn{2}{|c|}{ Cas } \\
\hline & & & $\begin{array}{c}\text { Confirmés en } \\
\text { laboratoire } \\
\mathrm{n}(\%)\end{array}$ & $\begin{array}{l}\text { Cliniques } \\
\mathrm{n}(\%)\end{array}$ \\
\hline Bactérie & $84(73)$ & $2588(78,4)$ & $2076(91,8)$ & $512(49,2)$ \\
\hline Salmonella & $47(40,9)$ & $2041(61,8)$ & $1776(78,5)$ & $265(25,5)$ \\
\hline Escherichia & $17(14,8)$ & $201(6,1)$ & $199(8,8)$ & $2(0,2)$ \\
\hline Clostridium botulinum & $3(2,6)$ & $3(0,1)$ & $3(0,1)$ & - \\
\hline Clostridium perfringens & $3(2,6)$ & $111(3,4)$ & $0(0)$ & $111(10,7)$ \\
\hline Listeria & $3(2,6)$ & $67(2)$ & $67(3)$ & - \\
\hline Campylobacter & $2(1,7)$ & $40(1,2)$ & $16(0,7)$ & $24(2,3)$ \\
\hline Staphylococcus & $2(1,7)$ & $23(0,7)$ & $2(0,1)$ & $21(2)$ \\
\hline Bacillus & $2(1,7)$ & $24(0,7)$ & - & $24(2,3)$ \\
\hline Shigella & $1(0,9)$ & $14(0,4)$ & $9(0,4)$ & $5(0,5)$ \\
\hline Cronobacter & $1(0,9)$ & $1(0)$ & $1(0)$ & - \\
\hline Autre & $1(0,9)$ & $16(0,5)$ & $3(0,1)$ & $13(1,3)$ \\
\hline Inconnue & $2(1,7)$ & $47(1,4)$ & - & $47(4,5)$ \\
\hline Virus & $17(14,8)$ & $422(12,8)$ & $52(2,3)$ & $370(35,6)$ \\
\hline Norovirus & $14(12,2)$ & $352(10,7)$ & $25(1,1)$ & $327(31,4)$ \\
\hline Virus de l'hépatite A & $2(1,7)$ & $27(0,8)$ & $27(1,2)$ & - \\
\hline Inconnu & $1(0,9)$ & $43(1,3)$ & - & $43(4,1)$ \\
\hline $\begin{array}{l}\text { Toxine ou produit } \\
\text { chimique }\end{array}$ & $6(5,2)$ & $88(2,7)$ & $12(0,5)$ & $76(7,3)$ \\
\hline $\begin{array}{l}\text { Intoxication par des } \\
\text { mollusques ou crustacés }\end{array}$ & $2(1,7)$ & $66(2)$ & $4(0,2)$ & $62(6)$ \\
\hline Intoxication à l'histamine & $2(1,7)$ & $8(0,2)$ & $8(0,4)$ & - \\
\hline $\begin{array}{l}\text { Intoxication par la toxine } \\
\text { contenue dans les crosses } \\
\text { de fougère }\end{array}$ & $1(0,9)$ & $9(0,3)$ & - & $9(0,9)$ \\
\hline Inconnu & $1(0,9)$ & $5(0,2)$ & - & $5(0,5)$ \\
\hline Autre & $4(3,5)$ & $128(3,9)$ & $121(5,4)$ & $7(0,7)$ \\
\hline Cyclospora & $3(2,6)$ & $121(3,7)$ & $121(5,4)$ & - \\
\hline $\begin{array}{l}\text { Intoxication par des levures } \\
\text { ou des champignons }\end{array}$ & $1(0,9)$ & $7(0,2)$ & - & $7(0,7)$ \\
\hline Inconnu & $4(3,5)$ & $75(2,3)$ & $0(0)$ & $75(7,2)$ \\
\hline Total général & $115(100)$ & $3301(100)$ & $2261(100)$ & $1040(100)$ \\
\hline
\end{tabular}

La Figure 2 illustre la proportion du nombre total d'enquêtes sur les éclosions d'origine alimentaire par année pour les trois agents pathogènes les plus fréquemment rapportées parmi les 106 éclosions d'origine alimentaire dont l'agent étiologique a été précisé. Au cours de la période d'étude, la proportion des éclosions à Salmonella a diminué de façon marquée en 2011 et 2012, période à laquelle elles ont été principalement rapportées par la Colombie-Britannique et par l'Agence. Au cours de la même période, l'Agence a observé une augmentation proportionnelle des éclosions liées à $E$. coli (données non illustrées). 
Figure 2 : Proportion du nombre total d'enquêtes sur les éclosions d'origine alimentaire signalées par l'entremise du Registre fédéral des éclosions par année selon l'agent étiologique, de 2008 à $2014(n=106)$

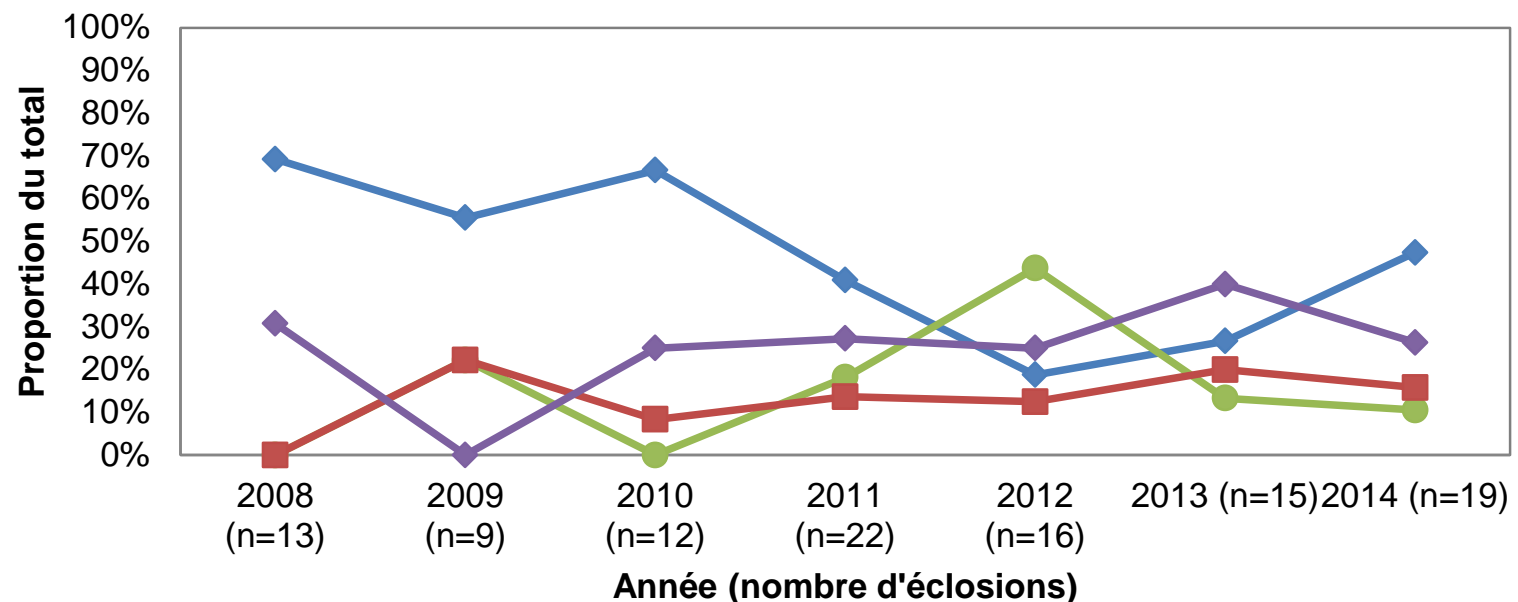

$\leadsto$ Salmonella $(n=47) \multimap-$ Escherichia $(n=17) \leadsto$ Norovirus $(n=14) \leadsto$ Autres $(n=28)$

\section{Gravité de la maladie}

Parmi les 3301 cas totaux associés aux éclosions d'origine alimentaire, 225 cas d'hospitalisation et 30 décès ont été signalés. Le nombre moyen de cas, d'hospitalisations et de décès associés à chaque agent pathogène varie grandement. Les agents étiologiques connus qui ont entraîné le plus grand nombre de cas par éclosion ont été Salmonella, Cyclospora, Clostridium perfringens et les intoxications par des mollusques ou des crustacés, avec une moyenne de $43,40,37$ et 33 cas par éclosion, respectivement.

Le Tableau 2 présente un résumé des données sur la gravité de la maladie, les hospitalisations et les décès. Regroupées, les infections dues à Salmonella et $E$. coli représentaient la majorité des cas d'hospitalisations (106 et 84, respectivement), mais la proportion des hospitalisations diffère grandement entre ces deux agents pathogènes. La proportion des hospitalisations a été, en moyenne, de $12 \%$ pour Salmonella et de $41,8 \%$ pour E. coli. Les intoxications causées par Clostridium botulinum et Listeria ainsi que l'intoxication à l'histamine avaient les proportions d'hospitalisations les plus élevées, avec $100 \%, 90 \%$ et $75 \%$ respectivement.

L'intoxication à Listeria était la cause la plus fréquente de décès liés à une éclosion, représentant 73,3\% du nombre total de décès (22 sur 30). Le taux de mortalité associé à Listeria a été de $32,8 \%$ parmi les cas liés à une éclosion. Les 22 décès liés à Listeria signalés dans le Registre fédéral des éclosions sont tous survenus au cours de la même éclosion et touchaient des personnes immunodéprimées et des personnes âgées se trouvant pour la plupart dans des hôpitaux ou des établissements de soins de longue durée au cours de leur période d'exposition 
Tableau 2 : Nombre d'éclosions d'origine alimentaire signalées par l'entremise du Registre fédéral des éclosions et nombre total de cas, d'hospitalisations et de décès selon l'agent étiologique, de 2008 à 2014 ( $n=115)$

\begin{tabular}{|c|c|c|c|c|}
\hline Agent étiologique & $\begin{array}{l}\text { Nombre moyen de } \\
\text { cas par éclosion } \\
\text { Moyenne (étendue) }\end{array}$ & $\begin{array}{l}\text { Nombre total } \\
\text { de cas } \\
\mathrm{n}(\%)\end{array}$ & $\begin{array}{c}\text { Hospitalisation } \\
\mathrm{n}\left(\%^{1}\right)\end{array}$ & $\begin{array}{l}\text { Décès } \\
\mathrm{n}\left(\%^{2}\right)\end{array}$ \\
\hline Bactérie & $30,8(1-1029)$ & $2588(78,4)$ & $205(15)$ & $30(1,2)$ \\
\hline Salmonella & $43,4(1-1029)$ & $2041(61,8)$ & $106(12)$ & $4(0,2)$ \\
\hline Escherichia & $11,8(1-30)$ & $201(6,1)$ & $84(41,8)$ & $3(1,5)$ \\
\hline Campylobacter & $20(10-30)$ & $40(1,2)$ & - & - \\
\hline Clostridium botulinum & $1,0(1-1)$ & $3(0,1)$ & $3(100)$ & $1(50)$ \\
\hline Clostridium perfringens & $37,0(28-54)$ & $111(3,4)$ & - & - \\
\hline Listeria & $22,3(5-57)$ & $67(2)$ & $9(90)$ & $22(32,8)$ \\
\hline Shigella & $14,0(14-14)$ & $14(0,4)$ & $2(14,3)$ & - \\
\hline Staphylococcus & $11,5(7-16)$ & $23(0,7)$ & $1(4,3)$ & - \\
\hline Bacillus & $12,0(11-13)$ & $24(0,7)$ & - & - \\
\hline Cronobacter & $1,0(1-1)$ & $1(0)$ & - & - \\
\hline Autre & $16,0(16-16)$ & $16(0,5)$ & - & - \\
\hline Inconnue & $23,5(4-43)$ & $47(1,4)$ & - & - \\
\hline Virus & $24,8(6-99)$ & $422(12,8)$ & $11(2,8)$ & - \\
\hline Norovirus & $25,1(7-99)$ & $352(10,7)$ & $1(0,3)$ & - \\
\hline Virus de l'hépatite $A$ & $13,5(6-21)$ & $27(0,8)$ & $10(47,6)$ & - \\
\hline Inconnue & $43,0(43-43)$ & $43(1,3)$ & - & - \\
\hline $\begin{array}{l}\text { Toxine ou produit } \\
\text { chimique }\end{array}$ & $14,7(2-62)$ & $88(2,7)$ & $7(26,9)$ & - \\
\hline Intoxication à I'histamine & $4,0(2-6)$ & $8(0,2)$ & $6(75)$ & - \\
\hline $\begin{array}{l}\text { Intoxication par des } \\
\text { mollusques ou crustacés }\end{array}$ & $33,0(4-62)$ & $66(2)$ & $1(25)$ & - \\
\hline $\begin{array}{l}\text { Intoxication par la toxine } \\
\text { contenue dans les crosses } \\
\text { de fougère }\end{array}$ & $9,0(9-9)$ & $9(0,3)$ & - & - \\
\hline Inconnu & $5,0(5-5)$ & $5(0,2)$ & - & - \\
\hline Autre & $32,0(7-85)$ & $128(3,9)$ & $2(1,6)$ & - \\
\hline Cyclospora & $40,3(11-85)$ & $121(3,7)$ & $2(1,7)$ & - \\
\hline $\begin{array}{l}\text { Intoxication par des levures } \\
\text { ou des champignons }\end{array}$ & $7,0(7-7)$ & $7(0,2)$ & - & - \\
\hline Inconnu & $18,8(6-41)$ & $75(2,3)$ & - & - \\
\hline Total général & $28,7(1-1029)$ & $3301(100)$ & $225(11,4)$ & $30(1)$ \\
\hline
\end{tabular}

${ }^{1}$ Les données indiquées représentent le nombre total par agent étiologique, avec la proportion de l'ensemble des cas pour chaque agent étiologique entre parenthèses. (Remarque : Les éclosions pour lesquelles il n’y a aucune valeur relativement aux hospitalisations et aux décès ont été exclues du dénominateur.)

${ }^{2}$ Les données sur le nombre d’hospitalisations sont manquantes pour 10 des 47 rapports sur les éclosions à Salmonella, pour 1 des 14 rapports sur les éclosions imputables au norovirus et pour 1 des rapports sur les éclosions imputables à Listeria, à Cronobacter, à l'hépatite A et aux intoxications par des mollusques et des crustacés. Les données sur le nombre de décès sont manquantes pour 8 des 47 rapports sur les éclosions à Salmonella, pour 2 des 14 rapports sur les éclosions imputables au norovirus et pour 1 des rapports sur les éclosions à Clostridium botulinum, à Bacillus cereus et à l'hépatite A.

\section{Durée}

Des données sur la date d'apparition des symptômes du premier cas ou du cas le plus récent étaient disponibles pour 94 des 115 (81,7 \%) éclosions d'origine alimentaire signalées par l'entremise du Registre fédéral des éclosions. La durée des éclosions variait de moins d'un jour à 1689 jours, les durées médiane et moyenne étant de 8 jours et 52,7 jours, respectivement. L'éclosion d'une durée de 1689 jours a été attribuée à une éclosion de $\mathrm{S}$. Enteritidis dans une même province, causée par des œufs et associée à 1029 cas confirmés en laboratoire.

\section{Exposition}

Dans l'ensemble, 44 éclosions d'origine alimentaire $(38,3 \%)$ et $60,8 \%$ des cas totaux liés à des éclosions d'origine alimentaire déclarées $(n=2006)$ sont survenus dans un milieu communautaire, tandis que 
37 éclosions $(32,2 \%)$ et $18,3 \%$ des cas totaux $(n=604)$ ont été associés à un établissement de services alimentaires. L'agent étiologique a été confirmé en laboratoire pour la majorité des éclosions d'origine alimentaire (96 cas sur 115 , soit $83,5 \%$ des cas).

Tableau 3 : Nombre d'enquêtes sur des éclosions d'origine alimentaire dont l'agent étiologique a été confirmé en laboratoire et signalées par l'entremise du Registre fédéral des éclosions, selon le milieu d'exposition et de transmission, de 2008 à 2014

\begin{tabular}{|l|c|c|c|}
\hline $\begin{array}{l}\text { Milieu d'exposition ou de } \\
\text { transmission }\end{array}$ & $\begin{array}{c}\text { Nombre } \\
\text { d'éclosions } \\
\mathrm{n}(\%)\end{array}$ & $\begin{array}{c}\text { Nombre d'éclosions } \\
\text { dont l'agent étiologique } \\
\text { a été confirmé en } \\
\text { laboratoire } \\
\mathrm{n}(\%)\end{array}$ & $\begin{array}{c}\text { Nombre total } \\
\text { de cas } \\
\mathrm{n}(\%)\end{array}$ \\
\hline Collectivité & $44(38,3)$ & $44(38,3)$ & $2006(60,8)$ \\
\hline $\begin{array}{l}\text { Établissement de services } \\
\text { alimentaires }\end{array}$ & $37(32,2)$ & $26(22,6)$ & $604(18,3)$ \\
\hline Événement privé & $14(12,2)$ & $10(8,7)$ & $24(0,7)$ \\
\hline Plus d'un milieu & $5(4,3)$ & $4(3,5)$ & $145(4,4)$ \\
\hline Milieu institutionnel résidentiel & $4(3,5)$ & $3(2,6)$ & $11(0,3)$ \\
\hline Milieu institutionnel non résidentiel & $4(3,5)$ & $2(1,7)$ & $1(0)$ \\
\hline Voyage à l'étranger & $1(0,9)$ & $1(0,9)$ & $33(1)$ \\
\hline Autre contexte & $2(1,7)$ & $2(1,7)$ & $411(12,5)$ \\
\hline Inconnu & $4(3,5)$ & $4(3,5)$ & $66(2)$ \\
\hline Total général & $\mathbf{1 1 5 ( 1 0 0 )}$ & $\mathbf{9 6 ( 8 3 , 5 )}$ & $\mathbf{3 3 0 1 ( 1 0 0 )}$ \\
\hline
\end{tabular}

La plupart des enquêtes ( 73 sur 115 , soit $63,5 \%$ des enquêtes) ont rapportés un aliment à la source de l'éclosion (données non illustrées). La viande a été la source la plus fréquemment signalée (26\%), suivi des œufs $(15,1 \%)$ et des légumes (13,7\%). Parmi les 47 éclosions à Salmonella, un aliment a été associé à l'éclosion dans 28 cas (59,6 \%); il s'agissait principalement d'œufs (39,3\%) et de viande (28,6\%). Douze des 17 cas d'éclosions imputables à $E$. coli $(70,6 \%)$ ont été associés à une source alimentaire précise et étaient principalement liés à des produits carnés (50\%). Les autres cas ont été associés à des légumes crus, à des noix et à des graines, à des produits laitiers et à des fruits. Enfin, 10 des 14 éclosions de norovirus ont été associées à des aliments précis, dont des fruits de mer, des fruits, des légumes et des aliments mixtes ou autres. Une éclosion de norovirus d'origine alimentaire a été associée à un manipulateur d'aliments malade.

Tableau 4 : Nombre d'aliments associés à une éclosion et proportion selon l'agent étiologique, de 2008 à 2014

\begin{tabular}{|l|c|c|c|c|c|c|}
\hline $\begin{array}{l}\text { Aliments associés à } \\
\text { l'éclosion }\end{array}$ & $\begin{array}{c}\text { Escherichi } \\
\mathbf{a} \\
\mathbf{n}(\%)\end{array}$ & $\begin{array}{c}\text { Norovirus } \\
\mathbf{n}(\%)\end{array}$ & $\begin{array}{c}\text { Salmonella } \\
\mathbf{n}(\%)\end{array}$ & $\begin{array}{c}\text { Autre } \\
\mathbf{n}(\%)\end{array}$ & $\begin{array}{c}\text { Inconnu } \\
\mathbf{n}(\%)\end{array}$ & $\begin{array}{c}\text { Total } \\
\mathbf{n}(\%)\end{array}$ \\
\hline Viandes & $6(50)$ & - & $8(28,6)$ & $5(27,8)$ & - & $19(26)$ \\
\hline Oeufs & - & - & $11(39,3)$ & - & - & $11(15,1)$ \\
\hline Légumes & $2(16,7)$ & $1(10)$ & $4(14,3)$ & $2(11,1)$ & $1(20)$ & $10(13,7)$ \\
\hline Fruits de mer & - & $3(30)$ & - & $5(27,8)$ & $1(20)$ & $9(12,3)$ \\
\hline Fruits & $1(8,3)$ & $2(20)$ & $3(10,7)$ & $1(5,6)$ & - & $7(9,6)$ \\
\hline Noix et graines & $2(16,7)$ & - & $1(3,6)$ & - & - & $3(4,1)$ \\
\hline Produits laitiers & $1(8,3)$ & - & - & - & - & $1(1,4)$ \\
\hline $\begin{array}{l}\text { Aliments mixtes ou } \\
\text { autres }\end{array}$ & - & $4(40)$ & $1(3,6)$ & $5(27,8)$ & $3(60)$ & $13(17,8)$ \\
\hline Total général & $\mathbf{1 2 ( 1 0 0 )}$ & $\mathbf{1 0 ( 1 0 0 )}$ & $\mathbf{2 8 ( 1 0 0 )}$ & $\mathbf{1 8 ( 1 0 0 )}$ & $\mathbf{5 ( 1 0 0 )}$ & $\mathbf{7 3 ( 1 0 0 )}$ \\
\hline
\end{tabular}




\section{Discussion}

Le présent rapport est le premier portrait national sur les éclosions d'origine alimentaire basé sur les données du Registre fédéral des éclosions. Les 115 éclosions de maladies entériques $(11,2 \%)$ signalées par l'entremise du Registre fédéral des éclosions étaient des éclosions d'origine alimentaire. Les agents étiologiques les plus souvent mis en cause étaient les bactéries Salmonella (40,9\%) et Escherichia (14,8 \%) ainsi que le norovirus (12,2 \%). Les données de surveillance provenant du Programme national de surveillance des maladies entériques (PNSME) reflètent aussi l'importance de la bactérie Salmonella en tant qu'agent étiologique des maladies entériques au Canada, cette bactérie ayant été l'agent pathogène (40,3\%) le plus fréquemment signalé au PNSME en 2013 (4). Il faut toutefois noter que le PNSME dresse un portrait plus large de la situation puisque les cas sporadiques et les cas d'infections contractées lors d'un voyage à l'étranger sont également inclus.

Le nombre total de maladies d'origine alimentaire sporadiques et contractées localement au Canada a aussi été estimé. Les cinq agents pathogènes les plus fréquents sont le norovirus (65,1\%). Clostridium perfringens (11\%) et Campylobacter spp. (8,4\%), Salmonella spp. non typhique $(5,1 \%)$ et Bacillus cereus $(2,3 \%)(1)$. Il est intéressant de noter les différences entre les cinq principaux agents pathogènes définis dans le cadre de cette étude et les agents pathogènes les plus souvent signalés dans le Registre fédéral des éclosions. Le sous-diagnostic et l'absence de confirmation en laboratoire de certaines infections, comme celles imputables à Campylobacter et à des toxines, ainsi que la sous-déclaration des cas et les pratiques d'enquête variables liées à ces agents pathogènes au Canada, pourraient expliquer pourquoi ces agents pathogènes n'occupent pas un rang aussi élevé dans le Registre fédéral des éclosions. Parmi les éclosions d'origine alimentaire survenues aux États-Unis en 2013 et dont l'agent étiologique était connu, le norovirus a été l'agent pathogène le plus courant $(44,8 \%)$, suivi de Salmonella $(32,3 \%)$ et de $E$. coli producteur de Shigatoxines (3,9\%) (4). La fréquence relative de ces agents pathogènes parmi les éclosions d'origine alimentaire diffère de celle observée au Canada, particulièrement pour le norovirus. Cela pourrait s'expliquer en partie par les différences qui existent entre les deux pays sur le plan des pratiques de déclaration et d'enquête.

Les proportions de cas hospitalisés imputables à Salmonella (12\%) et au norovirus (0,3\%) dans le Registre fédéral des éclosions étaient relativement proches de celles observées aux États-Unis $(17,5 \%$ et 0,7 \%, respectivement) (5). La proportion de cas hospitalisés était plus élevée pour la bactérie $E$. coli que pour Salmonella tant au Canada (41,8 \%) qu'aux États-Unis (33,3\%). Aucune comparaison entre les données sur les taux de mortalité dans le Registre fédéral des éclosions et les données recueillies aux États-Unis n'a pas pu être effectuée en raison du faible nombre de décès observés. La plupart des éclosions d'origine alimentaire signalées dans le Registre fédéral des éclosions étaient attribuables à un agent étiologique confirmé en laboratoire; toutefois, le rapport entre les cas confirmés en laboratoire et les cas cliniques était beaucoup plus élevé pour les éclosions bactériennes que pour les éclosions virales ou liées à une toxine ou un produit chimique. Cela est probablement imputable aux différences dans la présentation et la gravité des symptômes lors des éclosions bactériennes comparativement à d'autres types d'éclosions et à la probabilité d'un dépistage subséquent. Cette différence pourrait également être liée à l'efficacité des méthodes de dépistage des agents pathogènes viraux par rapport aux agents bactériens.

La plupart des éclosions d'origine alimentaire sont survenues au sein d'une collectivité ou d'un établissement de services alimentaires. La viande a été la source d'éclosion la plus fréquemment signalée (19 cas sur 73, soit $16,5 \%$ des cas), suivi des œufs (11 cas sur 73 , soit $15,1 \%$ des cas) et des légumes (10 cas sur 73 , soit $13,7 \%$ des cas). II est intéressant de noter qu'une proportion légèrement inférieure d'éclosions dont la source était connue a été signalée par les États-Unis (46 \% comparativement à 63,5\% dans le Registre fédéral des éclosions); le poisson était l'aliment le plus fréquemment signalé $(23,8 \%)$, suivi par les mollusques (11\%), le poulet (10\%) et les produits laitiers (10\%) (5).

L'avantage du Registre fédéral des éclosions est qu'il fournit une source de données permettant de suivre la fréquence et les tendances des maladies entériques et d'origine hydrique et alimentaire à l'échelle nationale. II offre aux partenaires à l'échelle fédérale et provinciale/territoriale un système leur permettant de récolter, de consulter et de résumer les données relatives aux éclosions de façon systématique et normalisée. Ces 
données ont été utilisées pour favoriser l'élaboration d'hypothèses dans le cadre des enquêtes sur les éclosions menées par l'Agence et par les autorités de santé publique participantes pour leurs propres systèmes de déclaration interne et leurs enquêtes sur les éclosions. Les données ont également servi à établir des rapports internes et à élaborer des projets menés au sein de l'Agence de la santé publique du Canada et de l'Agence canadienne d'inspection des aliments.

Plusieurs limites ont été identifiées sur le plan de l'analyse des données du Registre fédéral des éclosions. Les critères et les délais en matière de déclaration sont établis par chaque province participante et peuvent varier grandement, ce qui peut influer sur l'exhaustivité des données rapportées. Certains territoires de compétence qui ont rendu obligatoire le signalement des éclosions ont établi des délais de signalement, alors que d'autres partenaires ne l'ont pas fait. De plus, aucune ligne directrice n'a été établie à l'échelle nationale à ce chapitre. Les pratiques de déclaration ont aussi été modifiées au fil du temps, les provinces s'adaptant à l'utilisation du Registre fédéral des éclosions dans leurs territoires de compétence, ce qui a pu entraîner un changement quant aux types d'éclosions signalées. De plus, puisque que l'Agence et seulement six provinces signalent les éclosions par l'entremise du Registre fédéral des éclosions, les résultats de l'analyse présentée ici ne sont probablement pas représentatifs à l'échelle nationale. Enfin, des problèmes liés à la qualité et à l'uniformité des données ont été identifiés. À titre d'exemple, l'interprétation des dates de début et de fin des enquêtes varie d'un territoire de compétence à l'autre et les données manquantes pour ces variables, ainsi que les nombreux autres champs non obligatoires, limitent les conclusions qui peuvent être tirées de ces aspects des enquêtes sur les éclosions.

Les efforts déployés dans l'avenir viseront l'amélioration de la représentativité à l'échelle nationale par la contribution d'autres provinces et territoires au Registre fédéral des éclosions. En raison des limites énumérées précédemment, la discussion se poursuivra entre les partenaires afin d'améliorer la qualité des données et la cohérence du système. Finalement, les champs de données existants font actuellement l'objet d'un examen et l'introduction de variables supplémentaires est prévue, ce qui améliorera la description et la caractérisation des éclosions.

\section{Conclusion}

Cette analyse constitue le premier portrait à l'échelle nationale basé sur les éclosions signalées par l'entremise du Registre fédéral des éclosions. Ce système favorise l'échange de renseignements et la collaboration entre les partenaires du domaine de la santé publique au Canada. De plus, il permet de dresser un portrait à l'échelle nationale des éclosions d'origine alimentaire. À mesure que d'autres territoires de compétence adopteront le système, les données gagneront en fiabilité et renforceront notre capacité nationale à suivre les tendances et à contribuer à l'élaboration de politiques et à la planification en santé publique.

\section{Remerciements}

Nous aimerions souligner les contributions de nos collègues du RCRSP et les épidémiologistes des provinces et des territoires qui ont participé à l'élaboration et à la mise à jour du Registre fédéral des éclosions, de même que le travail assidu et l'engagement des professionnels canadiens de santé publique qui mènent avec diligence des enquêtes sur les éclosions de maladies entériques dans leurs territoires de compétence.

\section{Conflit d'intérêts}

Aucun.

\section{Financement}

Le financement de ce projet a été assuré par l'Agence de la santé publique du Canada. 


\section{Références}

(1) Thomas MK, Murray R, Flockhart L, Pintar K, Pollari F, Fazil A, et al. Estimates of the burden of foodborne illness in Canada for 30 specified pathogens and unspecified agents, circa 2006. Foodborne Pathog Dis. 2013 Jul;10(7):639-48.doi: 10.1089/fpd.2012.1389.

(2) Thomas MK, Murray R, Flockhart L, Pintar K, Fazil A, Nesbitt A, et al. Estimates of Foodborne Illness-Related Hospitalizations and Deaths in Canada for 30 Specified Pathogens and Unspecified Agents. Foodborne Pathog Dis. 2015 Aug 10. [Diffusion en ligne avant l'impression.]

(3) Reingold AL, Outbreak investigations--a perspective. Emerg Infect Dis. 1998 Jan-Mar;4(1): 21-27. doi: 10.3201/eid0401.980104PMCID: PMC2627653.

(4) Public Health Agency of Canada. National Enteric Surveillance Program Annual Summary 2013. 2015. Publication à venir. http://publications.gc.ca/site/fra/9.507317/publication.html

(5) Centers for Disease Control and Prevention (CDC). Surveillance for foodborne disease outbreaks, United States, 2013, Annual Report. Atlanta, GA: US Department of Health and Human Services, CDC; 2015. 